\title{
A Fast Maximum Likelihood Method for Improving AMCW Lidar Precision Using Waveform Shape
}

\author{
J. P. Godbaz*, M. J. Cree, A. A. Dorrington and A. D. Payne \\ Department of Engineering, University of Waikato, New Zealand \\ *Email: jpg7@waikato.ac.nz
}

\begin{abstract}
Amplitude Modulated Continuous Wave imaging lidar systems use the time-of-flight principle to determine the range to objects in a scene. Typical systems use modulated illumination of a scene and a modulated sensor or image intensifier. By changing the relative phase of the two modulation signals it is possible to measure the phase shift induced in the illumination signal, thus the range to the scene. In practical systems, the resultant correlation waveform contains harmonics that typically result in a non-linear range response. Nevertheless, these harmonics can be used to improve range precision. We model a waveform continuously variable in phase and intensity as a linear interpolation. By approximating the problem as a Maximum Likelihood problem, an analytic solution for the problem is derived that enables an entire range image to be processed in a few seconds. A substantial improvement in overall RMS error and precision over the standard Fourier phase analysis approach results.
\end{abstract}

\section{INTRODUCTION}

Amplitude Modulated Continuous Wave (AMCW) lidar systems use the Time-Of-Flight (TOF) principle to determine the range to objects in a scene. By measuring the phase offset in modulated scene illumination, the TOF, thus the range to objects in the scene is determined. Heterodyne lidar systems work by modulating the illumination at a high frequency and modulating a sensor or image intensifier at a slightly different frequency. The two signals are gain mixed, which results in a beating signal at a much lower frequency, with the phase offset of the beat signal proportional to the range to the object. Conventionally, Fourier analysis of the sampled beat signal is used to produce range data.

In practice, many systems use non-sinusoidal modulation signals [1]-[3]. In this case the recorded signal contains harmonics as the beat waveform is actually the correlation of the illumination and sensor modulation waveforms. In quadrature based systems this results in aliasing, requiring complex calibration or modulation techniques in order to produce linear range measurements [4]. In this paper, we show that flexible systems that can take a larger number of samples per beat can use this harmonic content to improve ranging precision.

In previous work [5] we applied a sparse spike train deconvolution technique to extract multiple returns within a pixel. In more recent work [6] we modelled the correlation (or beat) waveform using two different models. The first was a parametric truncated-triangle model and the second was an interpolation based method we called the Base-Ratio method.

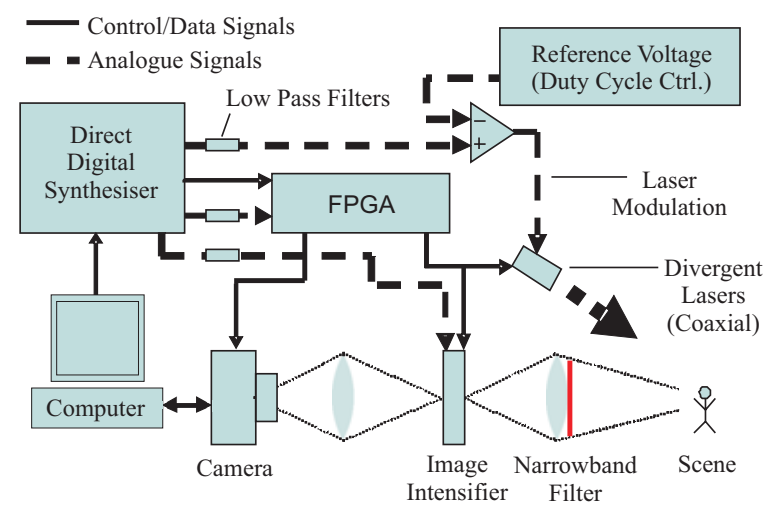

Fig. 1. University of Waikato Full-Field Lidar System

The new algorithm analytically calculates the phase offset of the waveform by maximising a weighted Gaussian likelihood function.

\section{A. The Waikato Full-Field Heterodyne Lidar System}

The University of Waikato Range-Imager [3] uses modulated laser light and an image intensifier to measure range (fig. 1). The system uses a Direct Digital Synthesiser (DDS) to generate sinusoids that are further processed to produce near rectangular laser and image intensifier modulation signals. The laser modulation signal is squared by passing it through a comparator, which compares the voltage to a user selectable reference voltage. By changing the laser duty cycle the shape of the correlation waveform can be changed, as shown in fig. 2 . This leads to changes in the precision and accuracy of the phase measurements. A CCD camera records the correlation waveform over time and the acquired signal is processed by a general purpose computer to calculate range. A narrowband filter is installed on the primary optics to limit ambient light.

There are three noise sources - Photon shot noise, readout noise and DDS jitter. Readout noise is insignificant compared to the others, so can be ignored. DDS jitter manifests as temporal waveform shape variation, i.e. the correlation waveform contains anharmonic frequencies.

\section{THEORY}

\section{A. Fourier Phase Analysis Approach}

For rectangular modulation waveforms most of the correlation waveform energy is concentrated in the fundamental. 


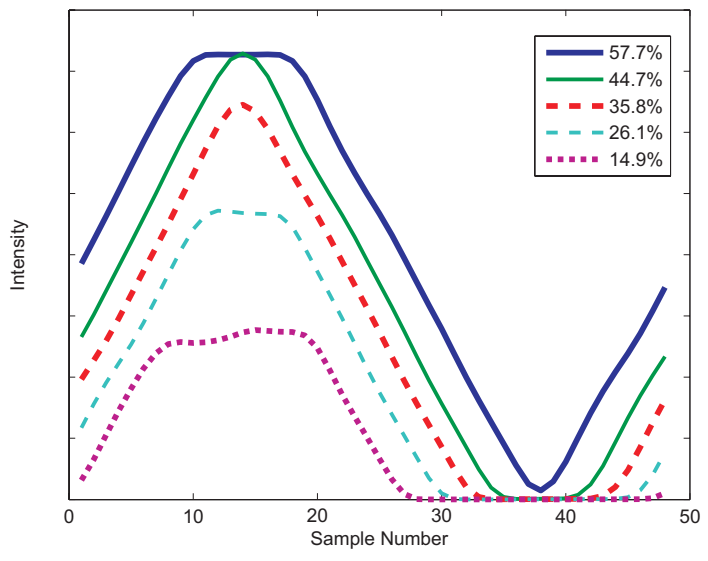

Fig. 2. Waveform Shape Versus Laser Duty Cycle.

A simple and reliable range measurement approach is to calculate the Fourier bin corresponding to the fundamental frequency. Since the entire Fourier Transform does not need to be calculated, this is bounded by $O(n)$ in Bachmann-Landau notation, where $n$ is the number of samples. This method is particularly reliable if there are a large number of samples as aliasing induced non-linearities are removed.

\section{B. Base-Ratio Model}

In ref. [6] we developed two models for the correlation waveform, allowing phase determination. One was a parametric piecewise truncated triangle model and the other was the Base-Ratio method, based on linear interpolation between a sampled waveform and a version translated by a single sample. The Base-Ratio model is fit to samples of the correlation waveform over time on a per pixel basis, and can be written as

$$
g[x]=I(\psi[x-\Upsilon]+\alpha \Delta \psi[x-\Upsilon]),
$$

where $g[x]$ is the estimated value of sample number $x, \psi$ is a cyclic waveform of infinite domain, $\Delta \psi[x]=\psi[x+1]-\psi[x]$ is the discrete derivative of the waveform, and $I$ is the relative intensity of the waveform. In order to allow a continuously phase variable waveform, the phase offset is composed of two summed components: $\Upsilon \in \mathbb{Z}$ is the coarse offset and $\alpha \in \mathbb{R}$, where $0 \leq \alpha \leq 1$, is the fine offset. The resultant phase offset is $\theta=2 \pi(\Upsilon+\alpha) / n$, where $n \in \mathbb{Z}^{+}$is the number of samples per cycle. This was posed as a Poisson Maximum Likelihood numerical optimisation problem in order to find $(I, \theta)$ for each pixel in a range image. This method resulted in a significant improvement in range precision and overall error versus the Fourier phase analysis approach, however, the numerical optimisation led to an extremely long processing time that was unviable for realistic applications. The structure of the problem also ignored the problem of background lighting passing through the band-pass filter (shown in fig. 1), which reduces the dynamic range of the system and causes constant offsets in the data.

\section{Proposed Model}

We extend eqn. 1 to account for background light, namely

$$
g_{2}[x]=I(\psi[x-\Upsilon]+\alpha \Delta \psi[x-\Upsilon])+\beta,
$$

where $g_{2}[x]$ is the estimated value of sample $x$ and $\beta$ is the amount of ambient light passing through the narrowband filter. We make the assumption that the ambient light is either nonvarying, or at a sufficiently high frequency that it can be modelled as a constant.

With sufficient light, Poisson distributed shot noise can be modelled as Gaussian distributed, $\sigma_{i}^{2}=\kappa v[i]+\epsilon_{i}^{2}$, where $\sigma_{i}^{2}$ is the power of the noise present in sample $i$, where $v[i]$ is sample $i$ of the recorded data, $\epsilon_{i}$ is readout noise and $\kappa$ is DN per photon. We do not currently know $\kappa$, although it could be determined. For this model $G_{2}=\left\{g_{2}[i] ; 0 \leq i<n\right\}$ and the recorded data $V=\{v[i] ; 0 \leq i<n\}$, we wish to find

$$
\underset{I, \theta}{\arg \max } P\left(G_{2} \mid V\right)
$$

which is equivalent to finding the maxima of the log-likelihood

$$
\begin{aligned}
L(I, \Upsilon, \alpha \mid V) & =c-\rho L_{r} \\
L_{r} & =\sum_{i=0}^{n-1} \omega[i]\left(g_{2}[i]-v[i]\right)^{2},
\end{aligned}
$$

where $c$ and $\rho$ are arbitrary constants, which we ignore, $L_{r}$ is a cost function and the weighting $\omega$ is the reciprocal of the variance of the noise at each point. Since $\kappa v[i] \gg \epsilon_{i}$, we approximate $\omega$ as

$$
\omega[i]=\frac{1}{v[i]} .
$$

strictly, $\omega[i]=g_{2}[i]^{-1}$ would be more correct, but is more difficult to optimise for. If the noise levels are particularly low, then the mismodelling of the linear interpolation can lead to errors, particularly at the waveform tail and leading edge, where the waveform is blurred due to the interpolatory convolution and is extremely heavily weighted due to eqn. 6 . Without further correction in the low noise situation we end up with poor results. We handle this by limiting the dynamic range of the weightings to $16: 1-$ any weightings above this threshold are truncated.

\section{Maximum Likelihood Solution}

The Maximum likelihood solution for $(I, \theta, \beta)$, given $V$ and $\Upsilon$ is found by setting the partial derivatives $\frac{\partial L_{r}}{\partial I}, \frac{\partial L_{r}}{\partial \alpha}$ and $\frac{\partial L_{r}}{\partial \beta}$ to zero and solving the resultant simultaneous equations. In order to simplify the equations, the following helper functions are defined:

$$
\begin{aligned}
\zeta_{y, z} & =\sum_{i=0}^{n-1} \omega[i] y[i] z[i], \\
\eta_{y} & =\sum_{i=0}^{n-1} \omega[i] y[i], \\
\Omega & =\sum_{i=0}^{n-1} \omega[i]
\end{aligned}
$$


for $y, z \in\{v, \psi, \Delta \psi\}$. In total 10 unique values are computed. Excluding the trivial case where $I=0$, then

$$
I=-\frac{\alpha_{d}}{\beta_{d}}, \alpha=\frac{\alpha_{n}}{\alpha_{d}}, \beta=\frac{\beta_{n}}{\beta_{d}},
$$

where $\alpha_{n}, \alpha_{d}, \beta_{n}$, and $\beta_{d}$ are defined by

$$
\begin{aligned}
\alpha_{n}= & \zeta_{v, \Delta \psi} \Omega \zeta_{\psi, \psi}-\Omega \zeta_{\psi, \Delta \psi} \zeta_{v, \psi}+\zeta_{\psi, \Delta \psi} \eta_{v} \eta_{\psi} \\
& -\zeta_{v, \Delta \psi} \eta_{\psi} \eta_{\psi}+\eta_{\Delta \psi} \eta_{\psi} \zeta_{v, \psi}-\eta_{v} \eta_{\Delta \psi} \zeta_{\psi, \psi} \\
\alpha_{d}= & \zeta_{\psi, \Delta \psi} \eta_{v} \eta_{\Delta \psi}-\zeta_{\psi, \Delta \psi} \Omega \zeta_{v, \Delta \psi}+\eta_{\Delta \psi} \eta_{\psi} \zeta_{v, \Delta \psi} \\
& -\eta_{v} \eta_{\psi} \zeta_{\Delta \psi, \Delta \psi}+\zeta_{v, \psi} \Omega \zeta_{\Delta \psi, \Delta \psi}-\zeta_{v, \psi} \eta_{\Delta \psi}^{2} \\
\beta_{n}= & -\eta_{\psi} \zeta_{\psi, \Delta \psi} \zeta_{v, \Delta \psi}+\eta_{\psi} \zeta_{v, \psi} \zeta_{\Delta \psi, \Delta \psi} \\
& +\eta_{\Delta \psi} \zeta_{v, \Delta \psi} \zeta_{\psi, \psi}-\eta_{\Delta \psi} \zeta_{\psi, \Delta \psi} \zeta_{v, \psi} \\
& +\eta_{v} \zeta_{\psi, \Delta \psi} \zeta_{\psi, \Delta \psi}-\eta_{v} \zeta_{\psi, \psi} \zeta_{\Delta \psi, \Delta \psi} \\
\beta_{d}= & \zeta_{\psi, \Delta \psi} \zeta_{\psi, \Delta \psi} \Omega-2 \zeta_{\psi, \Delta \psi} \eta_{\Delta \psi} \eta_{\psi}-\zeta_{\psi, \psi} \Omega \zeta_{\Delta \psi, \Delta \psi} \\
& +\zeta_{\psi, \psi} \eta_{\Delta \psi} \eta_{\Delta \psi}+\eta_{\psi} \eta_{\psi} \zeta_{\Delta \psi, \Delta \psi}
\end{aligned}
$$

Despite the apparent complexity, the above is still computationally simpler than trying to optimise eqn. 2 numerically.

The cost function can also be calculated as

$$
\begin{aligned}
L_{r}= & I^{2} \zeta_{\psi, \psi}+2 I^{2} \alpha \zeta_{\psi, \Delta \psi}+2 I \beta \eta_{\psi} \\
& -2 I \zeta_{v, \psi}+I^{2} \alpha^{2} \zeta_{\Delta \psi, \Delta \psi}+2 I \alpha \beta \eta_{\Delta \psi} \\
& -2 I \alpha \zeta_{v, \Delta \psi}+\beta^{2} \Omega-2 \beta \eta_{v}+\zeta_{v, v},
\end{aligned}
$$

which allows one to compare the likelihoods of several different coarse phase bins.

There are several ways in which the problem can be structured once we have this system of equations. One way is to explicitly enumerate all the possible values of $\Upsilon$ and calculate estimated values for $I$ and $\theta$ for each, then choose $\Upsilon$ with minimum $L_{r}$. This naive algorithm is $O\left(n^{2}\right)$ and wastes a large amount of time calculating estimates for improbable coarse alignments. However, if the noise level is sufficiently low a more informed choice can be made. Herein we choose to use the fundamental Fourier bin to find the two nearest phase bins. In most cases only one of the phase bins produces valid values, if both are valid the smallest $L_{r}$ is chosen, if neither is valid then the Fourier phase is used. In practice, with low noise levels, one is always valid. This results in an algorithm that is $O(n)$, although a constant factor slower than the Fourier analysis approach.

We do not explicitly constrain the values of $I$ and $\alpha$ because in low to moderate noise situations sensible values tend to be obtained. In high noise cases it is possible to massively overfit the data resulting, for example, in large negative values of $\alpha$.

\section{Methodology}

A flat board was placed in front of the range-imager and several sequence of beat cycles were captured at different laser modulation duty cycles at $20 \mathrm{MHz}$ modulation frequency, 48 samples per beat, 1 beat per second. The correlation waveform was sampled and then convolutionally blurred in order to simulate a worst case interpolation scenario. Range measurements were produced by post-processing these data using the Fourier analysis method, Base-Ratio method and the

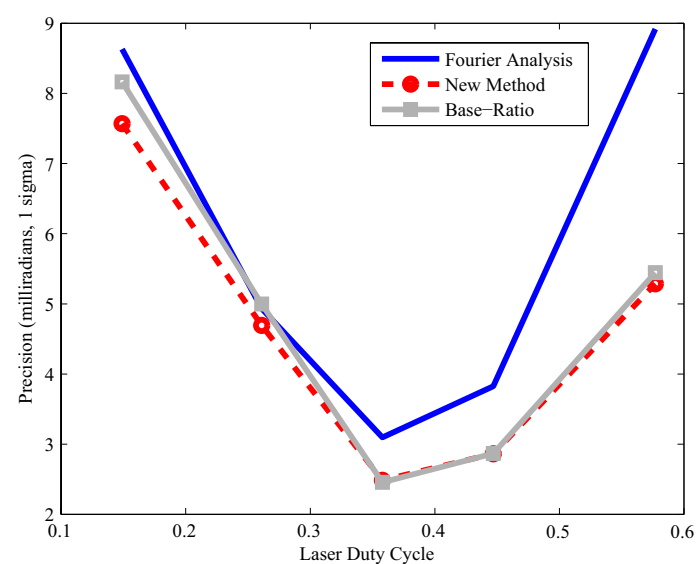

Fig. 3. Phase Measurement Precision

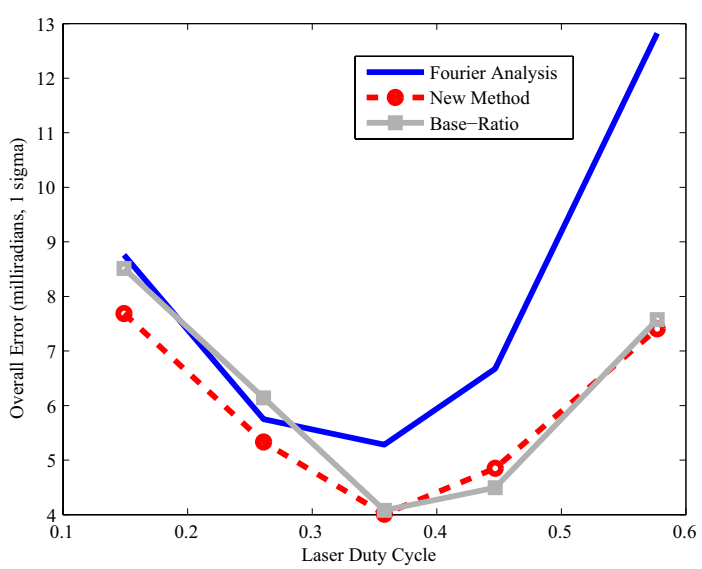

Fig. 4. Overall Phase Measurement Error (including systematic and random components)

new ML method. Precision was estimated by taking the sample standard deviation of each pixel's range measurements over time. Since the number of frames per cycle was sufficiently high to avoid aliasing, a range model was created by taking the Fourier analysis data, averaging it over time and then blurring it in the complex domain using a $\sigma=5$ pixels Gaussian blur. This model was then considered to be an accurate estimate of the actual range. The overall error was then calculated as the RMS error of the range measurements versus this model. Fig. 5 was produced by taking the sampled $35.8 \%$ duty cycle beat waveform, adding varying amounts of Poisson distributed noise and then taking range measurements via the Fourier and new ML approach.

\section{RESULtS}

Precision results in fig. 3 show a clear improvement in precision in the case of high duty cycles, with little improvement at low duty cycles. A contributing factor is the more complex shape of the lower duty cycle waveforms. Out of the five 


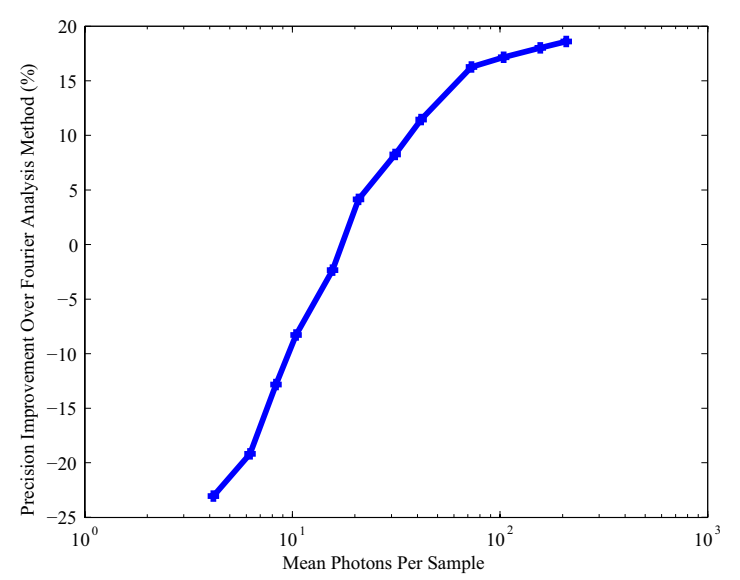

Fig. 5. Improvement in phase precision versus mean number of photons per sample for the new method versus Fourier analysis. Results generated via simulation from sampled $35.8 \%$ laser duty cycle correlation waveform.

different duty cycles sampled, $35.8 \%$ was clearly the best in all cases. Comparing overall error (fig. 4) to precision indicates an increase in systematic error components as the duty cycles increase. It is not due to simple offsets in the data and may be related to noise properties.

A simulation (fig. 5) shows how the amount of light collected affects the precision improvement. Low mean Poisson distributions are leptokurtic, thus poorly modelled by Gaussian distributions - hence below about 20 photons per sample, there is no precision improvement.

\section{A. Discussion}

The expected squared phase error is

$$
\sigma_{e}^{2}=E\left[(x-\mu)^{2}\right]=\int_{-\pi}^{\pi} x^{2} P(\mu+x \mid V) d x,
$$

where $\mu$ is the actual phase and $P(\mu+x)$ is the probability of producing an output phase $\mu+x$, given input data $V$ using correct Poisson statistics. Due to the structure of the algorithm it is very difficult to directly minimise this in order to generate an ideal waveform. There are also several physical constraints that make it difficult to generate an arbitrary waveform, for example, the correlation waveform is the convolution of an image intensifier modulation signal, a laser modulation signal and the impulse responses of both devices. This means that waveforms with substantial high frequency content are not possible - in our current setup we are limited to truncatedtriangle waveforms.

For the Fourier analysis method we know that precision is inversely proportional to the square root of the SNR. If we assume that Poisson distributed shot noise is the only noise source, then the noise power is proportional to the total integrated light intensity. We can calculate the signal strength as the absolute value of the fundamental Fourier bin giving

$$
\text { precision } \propto \frac{\sqrt{\sum_{i=0}^{n-1} v[i]}}{\left|\sum_{i=0}^{n-1} v[i] e^{-2 j \pi \frac{i}{n}}\right|} .
$$

However, since we are limited to rectangular modulation this becomes

$$
\text { precision } \propto \frac{1}{\sqrt{x} \operatorname{sinc} x}
$$

where $\{x \in \mathbb{R} ; 0 \leq x \leq 1\}$ is the duty cycle. This is a convex function with a global minimum at a $37.1 \%$ duty cycle that very closely matches the curve of the results in fig. 3 . Since this relationship is separable, this means that in the Fourier analysis case $37.1 \%$ is the optimum duty cycle for both the laser duty cycle and intensifier duty under all circumstances.

However, this does not necessarily hold when other noise sources are taken into account and when the new method is analysed. From an intuitive perspective on overall phase information content, $44.7 \%$ might have been expected to have the greatest overall phase information content as the sloped region could be considered to be a superset of the sloped region of any other waveform. Correct determination of the factors that influence the precision of the new method requires further analysis, although it appears to follow the general trend of the precision of the Fourier analysis method.

\section{CONCLUSION}

The algorithm substantially improves overall RMS error and precision across a range of laser duty cycles versus the standard Fourier analysis method, also performing slightly better than the Base-Ratio fitting method. The measurement precision depends upon the laser duty cycle, which can be optimised based on the parameters of the intensifier modulation using a simple relationship. Because the new method does not require numerical optimisation, it is over two orders of magnitude faster than the original Base-Ratio method.

\section{ACKNOWLEDGMENT}

J. P. Godbaz acknowledges the assistance of a New Zealand Tertiary Education Commission Top Achiever Doctoral Scholarship. This work is supported by the University of Waikato Strategic Fund.

\section{REFERENCES}

[1] S. Hsu, S. Acharya, A. Rafii, and R. New, "Performance of a time-offlight range camera for intelligent vehicle safety applications," Advanced Microsystems for Automotive Applications 2006, 2006, pp. 205-219.

[2] R. M. Schwarte, "Breakthrough in multichannel laser-radar technology providing thousands of high-sensitive lidar receivers on a chip," in Laser Radar Techniques for Atmospheric Sensing, Proceedings of the SPIE vol. 5575,2004 , pp. 126-136

[3] A. A. Dorrington, M. J. Cree, A. D. Payne, R. M. Conroy, and D. A. Carnegie, "Achieving sub-millimetre precision with a solid-state fullfield heterodyning range imaging camera," Measurement Science and Technology, vol. 18, no. 9, July 2007, pp. 2809-2816.

[4] A. D. Payne, A. A. Dorrington, M. J. Cree, and D. A. Carnegie, "Improved linearity using harmonic error rejection in a full-field range imaging system," Three Dimensional Image Capture and Applications, Proceedings of the SPIE vol. 6805, no. 1., 2008, pp. 68050D.

[5] J. P. Godbaz, M. J. Cree, and A. A. Dorrington, "Mixed pixel return separation for a full-field ranger," in Proceedings of Image and Vision Computing New Zealand '08, 2008, pp. 1-6

[6] J. P. Godbaz, "Multiple return separation for a full-field ranger via continuous waveform modelling," in Image Processing: Machine Vision Applications II, Proceedings of the SPIE vol. 7251, 2009, pp. 72510T 